\title{
A Case Study: An ICT Technology in Enterprise Integration
}

\author{
Jozef B. Lewoc ${ }^{1}$, Antoni Izworski ${ }^{2}$, Slawomir Skowronski ${ }^{3}$ and \\ Antonina Kieleczawa ${ }^{4}$ \\ ${ }^{1}$ BPBiT Leader, Powst. Sl. 193/28, 53-138 Wroclaw, Poland, \\ ${ }^{2}$ Wroclaw University of Technology, Wyb. Wyspianskiego 27, 50-370 Wroclaw, Poland, \\ ${ }^{3}$ Wroclaw University of Technology, Wyb. Wyspianskiego 27,50-370 Wroclaw, Poland, \\ ${ }^{4}$ Institute of Power System Automation, ul. Wystawowa 1,51-618 Wroclaw, Poland,
}

\begin{abstract}
An approach to development of pioneering Information and Communication Technology large-scale industrial enterprise integration technology is described for a case study of Computer Integrated Manufacturing and Management systems (CIMMs). The paper presents some experience in large-scale systems and the current state of art for the domain. A feasible approach is proposed, based on prospective user expectations and the system development with use of so called first thread on which the standards facilitating development of subsequent systems should be developed and people acquainted with both manufacturing and management problems should be educated. There is also depicted some research work intended for facilitating design and implementation of CIMMs. The approach proposed is particularly suitable for big and medium manufacturing plants
\end{abstract}

Keywords: Computer aided manufacturing, management, working media, environment, control, monitoring.

\section{BACKGROUND}

Large-scale and very large-scale systems have become a very serious social concern. Some people discuss even of the global systems. But, if one looks at normal industrial enterprises, the situation seems to be rather poor: on one hand, there are Computer Integrated ManufacturinG systems (CIMGs) developed by companies specialised in automation of industrial processes and Computer Integrated ManagemenT systems (CIMTs) systems developed by other companies, involved in automation of managerial activities. CIMGs and CIMTs are developed on heterogeneous hardware and software and, usually, the suppliers do not provide effective interconnections between their systems.

During a few past years, the problems were discussed with several dozen representatives of the case study country (Poland) (the Country), especially with Chief Working Media Engineers of metallurgical plants. The representatives were interested very much in development of Computer Integrated Manufacturing and Management systems (CIMMs) combining CIMGs and CIMTs, and believed that such systems are the necessary basis for optimum running of their enterprises through exact manufacturing cost assessment (costs per product or a production lot), enterprise-wide troubleshooting, optimisation of production plans, monitoring of implementation of new technologies, running the enterprise on the basis of some objective criteria, etc. Such enterprise operation optimisation seems to be a condition sine qua non for successful activity of their enterprises in the era of free market and the very competitive world (Horacek 2005).

Furthermore, many representatives did not believe that CIMMs were not available in the world computer market; the exemplary functions enumerated above were so obvious and interesting for them that they said CIMMs had to be available in some well developed countries.

Surely, they were wrong: within the offered product ranges of the major CIMT systems (e.g. IBM 2007; IB 2007; ICL 2007; IFS 2007), there are no complex solutions covering both management and manufacturing domain though some claim that they provide software for the industry. On the other hand, no information was found of companies or university centres involved in actual manufacturing control systems that offer complex solution covering the manufacturing and management domains. Most probably, the reason for such situation, severely inappropriate from the point of view of possible industrial users, is that the big manufacturers and, perhaps in a lesser extent, the academies involved in manufacturing are afraid of entering into the management domain and vice versa, because of lack of people of the necessary expertise in both areas. 


\section{METHODOLOGY AND DATA}

\section{Foreword}

CIMMS are, for sure, large-scale and novel systems. The authors, who have been involved in design and development of such systems know two basic methodologies in this respect: from bottom to top (bythinking) and from top to bottom (by-organising). The first one consists in safe and meek approach to system design - starting from possibly small part of the object under design, gathering experience and, at the end, developing the general concept and standards for next projects. The other methodology starts from the general concepts, standards and tries to implement them in the systems.

University of Cambridge Cavendish Laboratory applied the by-thinking approach for years. Example: their Radioastronomy Centre led by Prof. Martin Ryle got budgets for several year projects: ca. pound sterling $5000,50000,500000$. The final project resulted in more than half objects in the sky atlas identified by $\mathrm{C}$ for Cambridge, the aperture super-synthesis method, "by-the-way" discovery of pulsars, knight title and Nobel prize. Jodrell Bank centre, realised by the other methodology, cost much more and produced much, much smaller results. Sir Martin (Ryle 1970) mentioned many failures of the by-organising methodology, e.g. Jumbo Jets that decreased throughput of the Heathrow airport instead of increasing it. We may add the operating system OS MVT, HDLC compared with CCITT link level and many more contemporary examples. Most often, the methodology was highly overpaid and, very often, unsuccessful.

In the domain of CIMMs-es, the by-organisation methodology is also being applied. The ANSI/ISA-95 committee is developing ISA-95 series of standards that defines the interfaces between enterprise activities and control actions (ISA95 2008, Brandl \& Owen 2008). Even a high level language B2MML to link management systems with manufacturing systems has been developed. In opinion of the authors, the lack of the earlier experience in CIMMs-es makes it very improbable that the results of this work will be useful for design and development of actual computer integrated manufacturing and management systems.

Therefore, a team of designers and research workers involved in design and investigation of future CIMMs (the Team) chose the by-thinking technology from the very beginning.

\section{Some history}

The leading designer of the team has been involved in CIMMs projects since late eighties when a project of complex automation of power generation and management for a new big Polish power plant was initiated. The initial design of the Team covered power generating unit monitors plus a common power plant database (Badel (Lewoc et al 1989)) for power generation services and power plant management purposes (ref. Fig. 1). However, due to the down economy period and low power demand in the case study country (Poland), the project was suspended for many years and the Team was disassembled.

At the turn of the eighties, a project of CIMM was initiated by the then biggest Polish manufacturer of household appliances. However, due to the economic and personal problems of the manufacturer, the project was abandoned on an early phase.

After the very much desired and positive political change in the case study country in 1989, the technology transfer process become possible in a high degree. However, due to the ill approach of the "technology providers" (e.g. in Wroclaw, the former capital city of case study ICT and computer automation, no local pioneer in the domains was employed by the technology providers on an important technical position and a theory was devised that no team in the case study country is capable of implementing large scale ICT systems) (Blach 1991, Han 2008, Izworski 2001, Lewoc 2005, 2006 a,b) and in spite of rather impressing results of the Team (Kiejna 1988, Lewoc et al. 1983, 1984, 1989, 1992, 2008, Sawicki et al. 1974, Wojsznis \& Lewoc 1971), this work was not resumed nor continued in any way.

\section{Initiated work}

To counteract the evidently negative consequences of the situation, it was decided that the Team will be re-assembled in a part enabling to perform design (up to the feasibility study level) and research work for novel large-scale ICT applications (Abramczyk et al. 2004, 2005, Izworski et al. 2003c, Kiejna et al. 1988, Lewoc et al. 1990, 1992, 2006a,b,c,d), including that directly connected with CIMMs (Izworski et al. 2003a,b,c, 2006, Lewoc et al. 2006c, 2007). The design and research problems have been solved basing on volunteer work of the Team members, though the Polish term: praca spoleczna (work for the society) seems to be much more adequate in this context.

The direct objective of such work is the mobilisation of prospective users by presentation of feasible novel solutions and facilitation of future design work by developing of solutions and tools intended to assist the 
design and operating processes that will occur in the future systems (at least with some significant probability) so that they may demand firmly the systems and solutions meeting their needs.

Another objective is to develop system solution needed to solve the problems disclosed. The financial resources available to the Team are much too small to think about implementation of actual CIMMs. But the Team believe that it is not important who will develop such systems, it is of the severe importance that this work is done. To develop design and research solutions, not too big financing is necessary. Therefore, the Team decided to look for such solutions and publish them, in particular, on prestigious events. This results in an significant benefit for the Team's work: The best verification of their ideas would be the implementation. But the implementation is not possible soon due to the lack of financial resources and, even more, due to the ill policy of so called technology providers. In such case, the verification of the solutions by people, at least in theory, on the very top of the world's science and technology is a very, very precious thing.

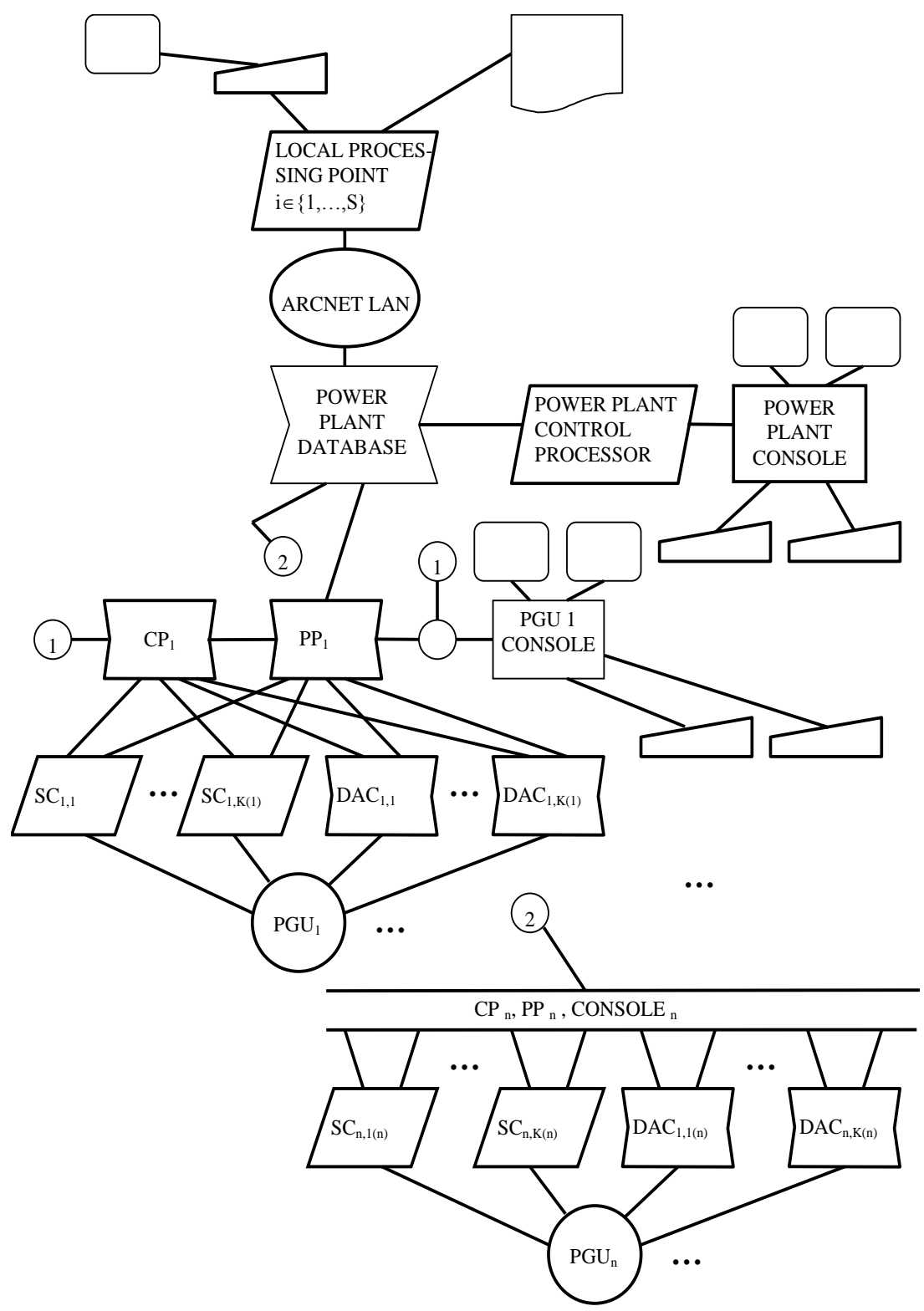

Fig. 1. Hardware architecture of Badel

Still another objective of the Team are performance and robustness evaluation of CIMMs. The team believes that, in spite of inconsiderate opinions of some prestigious event referees, this research work may result in definite benefits for the design work. 


\section{Approach to CIMM design}

\section{The goals}

The bases for development of the present CIMM design approach include the findings of the two early projects mentioned in Some History and conclusions of several dozen discussions held with representatives of various case study country plants, in particular metallurgical manufacturing plants. In most cases, the representative of the plant was the Head of the Media Department.

For the initial version of CIMM, the following rough goals have been defined:

The utilitarian goal:

To develop and implement the first thread (i.e. possibly simple set of tools necessary to implement a complete task in the network) on the CIMM network enabling a pilot interconnection between the CIMGs and CIMTs of an exemplary manufacturing enterprise.

The scientific goal of the first implementation is:

To develop standards and tools for design and implementation of CIMMs-ses for industrial enterprises.

The overall goal:

To provide bases for optimum operation of the enterprise upon some objective criteria.

\section{The first thread}

The approach to development of CIMMs-es was described by Izworski, 2006. The general organisation of any CIMM system could be planned only in a very general way now. Therefore, it is not reasonable to design a solution for all possible CIMM problems at the very beginning of the design process since it would require a lot of unnecessary and expensive work. Thus, the first thread in the CIMM system, i.e. the minimum set of tasks needed for development a interconnection of the CIMGs and CIMTs useful an profitable for the enterprise involved, should be defined, feasible to be designed, worked out and implemented by a finite team. Basing on these experience and standards worked out, it should be possible to design and implement further CIMM threads.

Especially for metallurgy plants, where the costs of working media exceed, in general, $20 \%$ of the overall manufacturing costs, the best candidate for the first CIMMs thread seems to be the Working Media Department which exists in each manufacturing enterprise. Basic evidence:

- high share of working media in total enterprise costs,

- similar problems in many enterprises,

- a lot of experience in the power industry CIMGs-es may be applied directly for CIMMs-es,

- power saving and sustainable energy problems are very important now what makes CIMMs-es even more attractive,

- the Department knowledge of all personnel in the enterprise is of importance for CIMMs development.

\section{System MEDIA}

\section{Basic variables}

The basic variables proposed for the system MEDIA (ref. Fig. 2) for a typical metallurgical plant are: For electric power: active and reactive power, voltage, current, energy; For gas: flow, calorific value; For fuel: weight; For water: flow, temperature; For steam: flow, temperature, pressure; For waste water: BOD, COD, heavy metals, suspended solids, oil content, ph, flow; For flue gas: flow, $\mathrm{CO}$ content, $\mathrm{SO}_{\mathrm{x}}, \mathrm{NO}_{\mathrm{x}}$.

\section{Hardware architecture of system Media}

The general structure of the system MEDIA is presented in Fig. 2. The basic reason for the proposed solution (a separate programmable controller (s) (PLC) for each medium in order that the design, programming and maintenance efforts are minimised. This structure is commonly used in the power industry and in many CIMGs-es and the type oh hardware should be selected on the basis of the most effective development of CIMMs-es. 


\section{Software architecture of system Media}

The system MEDIA software consists basically of the monitoring system software (the controllers' software are standard process variable acquisition and primary processing programs). The operating system should be selected taking into consideration its dependability features.

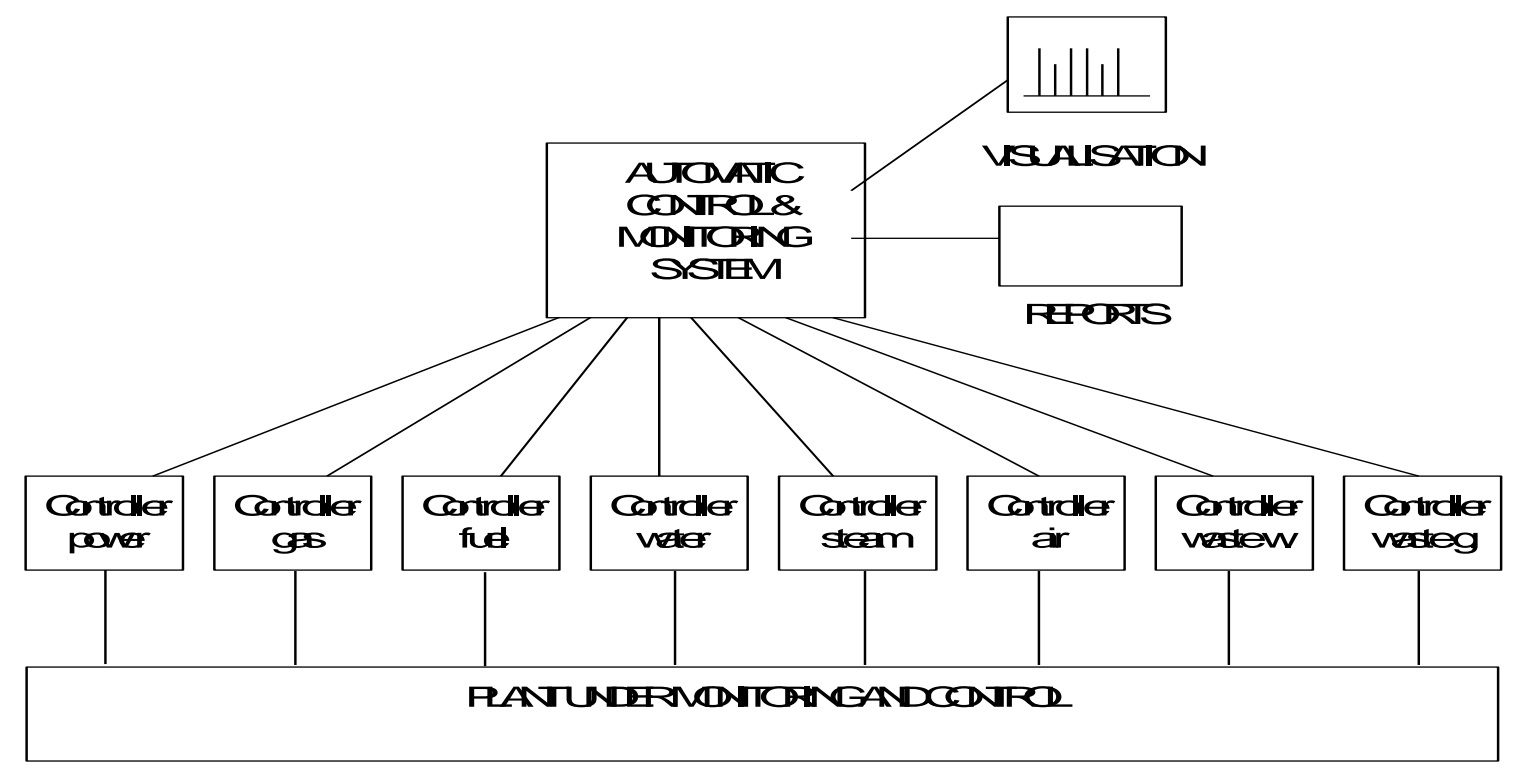

Fig. 2. Architecture of system MEDIA

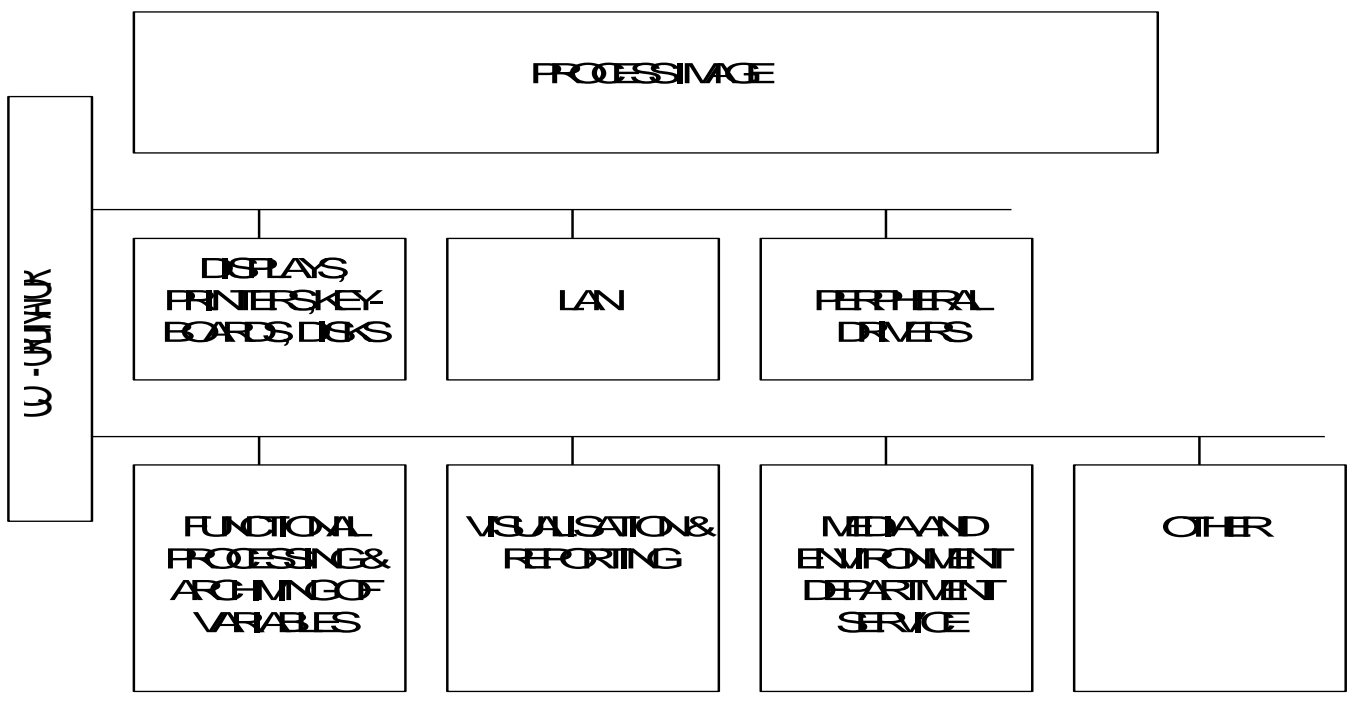

Fig. 3. System Monos software architecture 


\section{The first thread}

The first thread through CIMMs under consideration is depicted in Fig. 4.

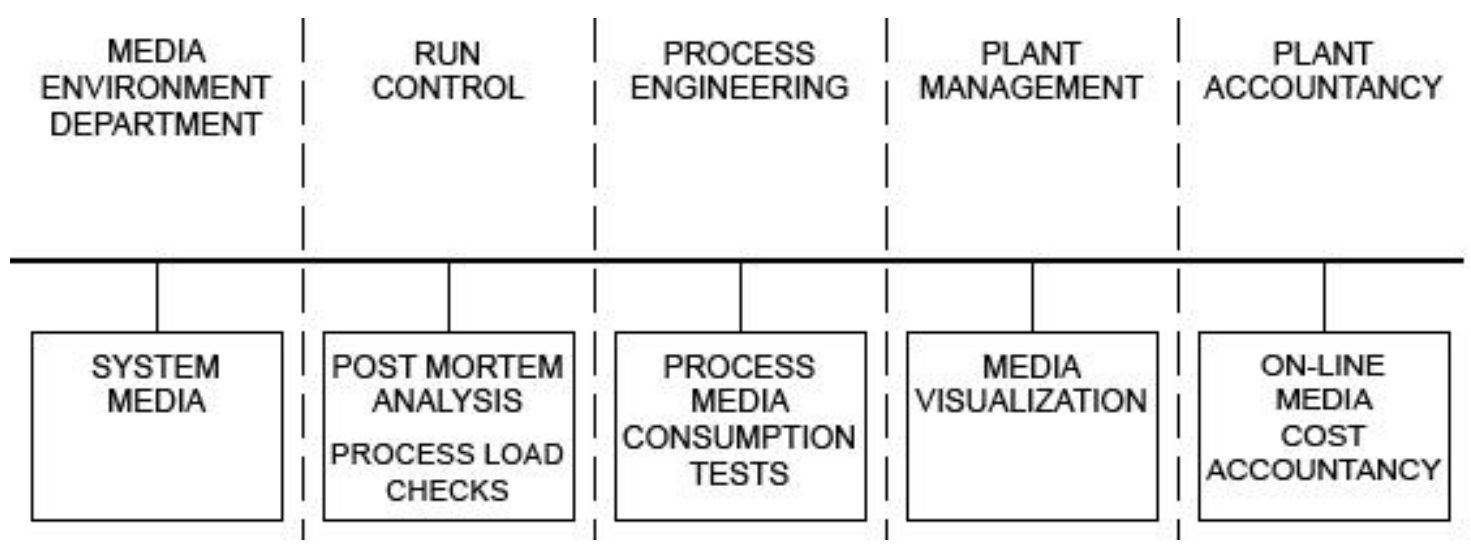

Fig. 4. The first thread through CIMMs

The first thread starts at the system MEDIA discussed hereinabove. It should be complemented of the post mortem analysis and process load/consumption tests, severely needed by the run control departments responsible for preparation of adequate run conditions, and of the process media consumption tests to evaluate process engineering designs, especially for new products. Interconnection between the system MEDIA and the plant management level should enable displaying of standard MEDIA screens for the managerial staff, thus increasing the observation possibilities of the latter. And, last but in no case least, the on line media cost accounting should be the first step towards cost accounting per a product. This does not require writing new accountancy systems; the idea is that the system MEDIA "sells" media in conformity with current readings of the variables under control, thus enabling on-line monitoring of media used and associating the costs with individual products or production batches.

\section{FINDINGS}

The Team defined successfully a feasible first thread through the CIMM system. Possible hardware and software structures were analysed and found useful for actual applications (Izworski et al. 2006, Lewoc et al. 2006d).

Some important aspects of CIMMs were investigated by research methods. In particular, the MEDIA system architecture was chosen on the basis of robustness assessment. To assess and compare robustness, the very basic function $\mu$ was applied (Doyle 1982). Many researchers claim that the $\mu$ function is hardly approximable (Cozman 1997, Ferreres 1999, Fu 1997, Maciejowski 1989). However, the Team found that digital systems may be analysed as composed of time lag components only of the transfer functions on the unit circle, where all values are eigen values. Due to that, calculations become rather simple and the $\mu$ function methods may be applied effectively. The team applied them for comparison of various MEDIA system topologies ; from the point of view of robustness, the star topology is the best one (Izworski \& Lewoc 2003a,c).

The Team applied also the methodology proposed by Lewoc 1990 for investigation of computer networks (in particular, those employing the TCP/IP protocol suite) that may be considered eligible for implementation of CIMMs. The basic references available (Robertazzi 2000, Surfozo 1999) do not present adequate methods for investigation of actual computer networks. The methodology proposed by Lewoc 1990 is based on investigation of so called closed loops which are an isomorphic mapping of actual virtual calls or datagram services (which are also some kinds of virtual calls implemented in higher network layers). For the closed loops, simple and straightforward approximations are done, leading to performance evaluation with a relative error less than some $5 \%$, as assessed by simulation and with a specially tailored internal network measuring tool.

The Team submitted some papers on applications of the approximate method to some networking conference. The referees' opinions seems to be very interesting: apply MV (2 times), the only way is to apply the random Markov chain method, it is known that no performance evaluation methods may be effectively applied in design. The "good advices" induce only a shame in a human being that the 
level of refereeing for prestigious events may be so poor. Nevertheless, the papers of the Team were published elsewhere, the Eurosim circles, known of high mathematical traditions and culture, being worth noting (Izworski \& Lewoc 2003b, Lewoc et al 2006c, 2007).

\section{CONCLUSIONS}

It is hard to believe that, considering the very low knowledge and experience level in CIMMs-es, the widely advertised work on the standards and language in the domain can accelerate development of CIMMs-es or make them more economic. On the contrary, earlier examples show that, usually, the top to bottom method applied for novel systems results in that they are seriously overpaid if successful at all.

CIMMs-es should be developed by the by-thinking method, safely acquiring the knowledge of the application and the experience needed to develop future applications.

CIMMS-es should be developed along the first thread beginning at the media department and leading to the accountancy department. Such thread is feasible with no need of replacing accountancy systems, by the way of standard customisation of well-known solutions.

The very basic robustness assessment methods, using the $\mu$ function, are directly applicable to comparative studies of digital systems described by time-delay members only.

Performance evaluation methods developed by the network designers themselves may be very useful tools, especially when the serious inutility of the closed queuing network is taken into account.

\section{References}

1. Abramczyk A., Izworski A. \& Lewoc J.B., A Case Study: Risks \& Challenges for Training Nurses in Diabetes Problems, Second IFIP International Summer School: Risks \& Challenges of the Network Society, Karlstad.

2. Abramczyk A., Lewoc J.B. and Izworski A., Nurse Training case study: e-training of nurses in diabetes problem, Nurse Education In Practice (Elsevier), Vol. 5, Issue 2, pp. 70-77, 2005

3. Abramczyk A., Lewoc J.B. and Izworski A., Nurse Training case study: e-training of nurses In diabetes problem, Nurse Education In Practice (Elsevier), Vol. 5, Issue 2, pp. 70-77, 2005

4. Bieleninik E., Lewoc J.B. \& Stanisz A. 1987. A Communication Network of Polish University Network, Technika Obliczeniowa Krajow Socjalistycznych, No. 22 (in Russian).

5. Blach L.K. et al. 1991. West-East Approaches of Automation: A Case Study. Cultural Aspects of Automation (IFAC), Krems.

6. Brandl D. \& Owen P. 2008. Available at: www.briconsulting.com/Files/2003-09\%20Cambridge-V03.ppt.

7. Cozman F. 1997, Robustness analysis of Bayesian networks with local convex sets of distributions, Uncertainty in Artificial Intelligence, Morgan \& Kaufmann, San Mateo.

8. Doyle J.C. 1982, Analysis of feedback systems with structured uncertainties, Proceedings of the Institution of Electrical Engineers, Part D, 129, 42-50.

ISA95 2008. Available at: www.isa.org/MSTemplate.cfm?MicrositeID =285\&CommitteeID=4747

10. Horacek P. et al. Editors 2005, Manufacturing Topic 5, $16^{\text {th }}$ IFAC World Congress, Prague.

11. Ferreres G. 1999, A Practical Approach to Robustness Analysis with Aeronautical Applications, Kluver/Plenum.

12. Fu M. 1997, The Real mu is Hardly Approximable, IEEE Trans. Auto. Control, vol. 42, no. 9, pp. 1286-1288.

13. Han M-W., Lewoc J.B., Izworski A., Skowronski S. \& Kieleczawa A. 2008, Power Industry Computer Control System Design and Implementation Problems: A Case Study of Poland. IFAC Congress, Seoul.

14. IBM 2007, Product Range, Available at: www.ibm.com.

15. IBS 2007, Product Range, Available at: www.ibs.se.

16. ICL 2007, Product Range, Available at: www.icl.org.

17. IFS 2006 Product Range, Available at: www.ifsworld.com.

18. Izworski A. et al. 2001 Some Aspects of Technology Transfer - A Case Study, SWIIS'01, IFAC, Vienna.

19. Izworski A. and Lewoc J.B. 2003a. Robust performance case study: Topology of system Media, Control Applications of Optimisation IFAC, Visegrad.

20. Izworski A. and Lewoc J.B.. 2003b. Approximate analytical performance modelling of a computer integrated manufacturing and management system. Mathematical Modelling IMACS, Vienna.

21. Izworski A. and Lewoc J.B. 2003c, Robustness Comparison of Enterprise Energy Distribution Systems of Various Topologies, Robust System Design IFAC, Milan.

22. Izworski A., Lewoc J.B. and Skowronski S. 2006. Development of Computer Integrated Manufacturing and Management Systems. Information Control Problems in Manufacturing IFAC, Saint Etienne.

23. Kiejna A. and Lewoc J.B. 1988, An Exemplary Implementation of Computer Technique for Schizophrenia Studies, The European Journal of Psychiatry, 2 No. 1.

24. Lewoc J. and Rozent M. 1975, An Operating System for Digital Simulator of Power Generating Unit, Future progress of Electrical Engineering, Prague.

25. Lewoc J.B. and Lanowska B. 1983, Microcomputer Data Display System for Power Industry Applications, Micro-P '83, Budapest.

26. Lewoc J.B. 1984, A microcomputer Data Acquisition and Retrieval System for Power Distribution Boards, Microelectronics 1984, Prague.

27. Lewoc J.B., Slusarska E. and Tomczyk A. 1989, A Distributed Data Base for Real-Time control / Monitoring of Power Plants, Distributed Data Bases in Real-Time Control IFAC/IFIP, Budapest.

28. Lewoc J.B. 1990, Performance Evaluation Problems for Actual Computer Networks, AMSE Monographs, Tassin.

29. Lewoc J.B., Rozent M., Saczuk I. 1992. The computing power and the computer

30. Lewoc J.B. 2005, A Case Study: Practical Ethical Aspects for Technology Transfer and Change In Computer Control System Development. IFAC Congress, Prague.

31. Lewoc J.B. et al.2006a, Automation, Control and Supervision of Combined Heat and Power Systems: A Case Study of Poland. International Stability through Automation '06, Prishtina IFAC. 


\section{A Case Study: An ICT Technology in Enterprise Integration}

32. Lewoc J.B., Izworski A. and Skowronski 2006b. Economic, Ethical and Cultural Aspects of ICT Change in Power System Automation: a Case Study of Poland International Stability through Automation '06, Prishtina IFAC,

33. Lewoc J.B., Izworski A. and Skowronski S. 2006c. An Internal Internet Traffic Modelling / Performance Measuring Tool. Mathematical Modelling IMACS. Vienna.

34. Lewoc J.B., Izworski A. and Skowronski S. 2006d. Alsis Case Study:computer integrated manufacturing and management systems. In; Applications of Large Scale Industrial Systems, Helsinki-Stockholm IFAC.

35. Lewoc J.B., Izworski A. \& Skowronski S. 2007. Performance Modelling of a Computer Integrated Manufacturing and Management System. Eurosim Congress, Ljubljana, 2007.

36. Lewoc J.B., Izworski A., Skowronski S. \& Kieleczawa A. 2008a, A Case Study: History of Polish Computer Applications in Power System Control. In IFIP World Computer Congress. Milano.

37. Maciejowski J.M. 1989, Multivariable Feedback Design, Addison-Wesley, Wokingham.

38. Robertazzi T.G. 2000, Computer Network and Systems. Queuing Theory and Performance Evaluation, Springer-Verlag.

39. Ryle M. 1970, Private message. Cambridge.

40. Sawicki J., Kowalski A.J. and Lewoc J. 1974, Implementation of the Automatic Data-Processing for the Power System Control in Poland, Data - Processing Conference, Nr 5.1.11, Madrid.

41. Surfozo R. 1999, Introduction to Stochastic Networks, Springer-Verlag.

42. Wikipedia 2008, B2MML. Available at: http://en.wikipedia.org/wiki/B2MML.

43. Wojsznis W. and Lewoc J. 1971, Metal Flow Control System in a Steel Mill, Production Process Automation in Black Metallurgy, Publication I/33, Zaporoze in Russian. 